\title{
Estudio de la concordancia en el recuento endotelial tras cirugía de catarata realizada por cirujanos noveles o expertos
}

\section{Study of concordance in endothelial cell count after cataract surgery performed by novice or expert surgeons}

\author{
Beatriz Alonso-Martín ${ }^{1 *}$, Juan C. Herranz-Heras ${ }^{1}$, Ignacio Cañas-Zamarra ${ }^{1}$, María Hernando-Portela ${ }^{1}$, \\ Elena Redondo-Martínez ${ }^{1}$, Rosa Ma. Sierra-Torres ${ }^{1}$, Agustín Gómez-de la Cámara ${ }^{2} y$ \\ Beatriz Sarmiento-Torres ${ }^{1}$ \\ ${ }^{1}$ Servicio de Oftalmología; ${ }^{2}$ Instituto de investigación i+12. Hospital Universitario 12 de Octubre, Madrid, España
}

\section{Resumen}

Objetivo: Evaluar la pérdida celular endotelial en pacientes operados mediante facoemulsificación de catarata no complicada, comparando datos de cirujanos noveles y cirujanos expertos. Método: Se realizó un estudio observacional y prospectivo en el que se evaluaron 50 ojos consecutivos aleatorizados en dos grupos de 25 ojos cada uno. Se realizó a cada ojo recuento de células endoteliales mediante microscopía especular preoperatoriamente y al mes de la cirugía. Los ojos con cataratas complicadas fueron excluidos. Resultados: La mayoría de los pacientes eran mujeres, con una edad media de 75.5 años. La disminución de la densidad celular al mes de la cirugía fue significativa tanto en ojos operados por cirujanos expertos como por cirujanos noveles, con una pérdida del $14.8 \%$ y el 15.6\%, respectivamente, sin diferencia estadísticamente significativa $(p=0.491)$. Además, la correlación entre la pérdida de ambos grupos fue de $0.58(p=0.0094)$. La pérdida celular en ojos operados por cirujanos noveles demostró una buena correlación (0.577) con la energía acumulada disipada del ultrasonido durante la facoemulsificación ( $p=0.0025)$, frente a los expertos, en los que no pudo demostrarse esta asociación. El total de energía acumulada disipada empleada por cirujanos noveles fue significativamente mayor $(p=0.045)$, un 63\% más que los expertos. Conclusiones: La diferencia en el recuento endotelial tras cirugía de catarata se demuestra asociada a la propia cirugía, con cifras similares para cirujanos noveles y expertos, y se observa una buena correlación entre ambos grupos. Teniendo en cuenta la pérdida de celularidad endotelial corneal, la seguridad del procedimiento realizado por cirujanos noveles es equivalente a la del realizado por expertos.

Palabras clave: Células endoteliales. Microscopía. Facoemulsificación. Catarata. Seguridad. Cirujano.

\section{Abstract}

Objective: To evaluate endothelial cell loss in patients after uncomplicated phacoemulsification comparing data from novice surgeons and expert surgeons. Method: An observational and prospective study was conducted in which 50 consecutive randomized eyes were evaluated in two groups of 25 eyes each. An endothelial cell count with specular microscopy was performed preoperatively and one month after surgery. Eyes with complicated cataracts were excluded. Results: Most of the patients were women, with a mean age of 75.5 years. The decrease in endothelial cell density one month after surgery was

Fecha de recepción: 24-05-2020 Fecha de aceptación: 12-05-2021 E-mail: Beatriz_alonso@live.com 0187-4519/๑ 2021 Sociedad Mexicana de Oftalmología. Publicado por Permanyer. Este es un artículo open access bajo la licencia CC BY-NC-ND (http://creativecommons.org/licenses/by-nc-nd/4.0/).
Disponible en internet: 01-09-2021 Rev Mex Oftalmol. 2021;95(5):193-202

www.rmo.com.mx 
significant both in eyes operated by expert surgeons and novice surgeons, with a loss of $14.8 \%$ and $15.6 \%$, respectively, with no statistically significant difference $(p=0.491)$. Furthermore, the correlation between both groups was $0.58(p=0.0094)$. Cell loss in eyes operated by novice surgeons showed a good correlation (0.577) with the cumulative dissipated energy during phacoemulsification ( $p=0.0025)$, contrary to the results obtained with expert surgeons where this correlation could not be demonstrated. The total cumulative dissipated energy used by novice surgeons was significantly higher $(p=0.045)$; $63 \%$ more than expert surgeons. Conclusions: Phacoemulsification resulted in significant corneal endothelial cell damage with similar figures for both groups, showing a good correlation between them. Concerning endothelial cell loss, the safety of the procedure performed by novice surgeons is equivalent to that performed by experts.

Keywords: Endothelial cell. Microscopy. Phacoemulsification. Cataract. Safety. Surgeon.

\section{Introducción}

La catarata senil es una enfermedad crónica asociada al proceso de envejecimiento, y es la principal causa de ceguera reversible en el mundo. Se estima que 285 millones de personas presentan algún grado de discapacidad visual, de los cuales el $33 \%$ se debe a cataratas ${ }^{1}$. Clínicamente se definen como la disminución de la agudeza visual provocada por la opacificación del cristalino y habitualmente se presenta de manera bilateral.

A edades menores de 50-55 años las prevalencias son bajas, del orden del $0.2-7 \%$, pero a partir de los 70-75 años las cataratas afectan al $40-60 \%$ de la población 0 más, según las series ${ }^{2}$. El paulatino ascenso de la esperanza de vida ha provocado un aumento sustancial de la prevalencia de cataratas, que afectan a una proporción creciente de la población.

La facoemulsificación con implante simultáneo de lente intraocular es la técnica con mejor coste-beneficio en términos de restauración de calidad de vida, y a día de hoy es la más utilizada en nuestro entorno. Esta técnica mecanizada de extracción del cristalino fue descrita por primera vez por Charles Kelman en 1967, basándose en la fragmentación y la aspiración de los fragmentos de la catarata mediante ultrasonidos, interviniendo en el interior del saco capsular a través de una incisión pequeña'.

Una de las principales complicaciones de la cirugía de cataratas es la pérdida de células endoteliales derivada de la técnica quirúrgica. El endotelio corneal está compuesto por una monocapa de células hexagonales en forma de mosaico que deriva de la cresta neural. Es la estructura de mayor importancia para preservar la transparencia de la córnea, condicionada sobre todo por su estado previo. El endotelio carece de capacidad proliferativa, pero en caso de daño endotelial, bien por causa fisiológica debido a la edad o por causa traumática como puede ser una cirugía ocular, el mosaico monocapa se restablece mediante migración y aumento de tamaño de las células vecinas a lo largo de los meses siguientes a la lesión, aumentando el coeficiente de variación del área celular (polimegatismo) y disminuyendo el porcentaje de células hexagonales (pleomorfismo).

En córneas jóvenes, la media de densidad celular se encuentra en torno a 3500 células $/ \mathrm{mm}^{2}$, con un coeficiente de variación que se aproxima a 0.25 y un $70-80 \%$ de células hexagonales. No se conoce con exactitud la densidad de células endoteliales necesaria para mantener la turgencia y la transparencia de la córnea, aunque se cree que entre 400 y 700 células $/ \mathrm{mm}^{2}$ podría ser el límite 3 .

El primer microscopio especular fue introducido por Maurice a finales de la década de 1960, siendo esta la modalidad semiológica más objetiva para evaluar clínicamente el endotelio corneal sin alterar su función ni su morfología, y evitando la posibilidad de transmisión de infecciones ${ }^{4-6}$.

Por los motivos expuestos, nos planteamos realizar este estudio cuyo objetivo primario fue evaluar la pérdida celular endotelial en pacientes sometidos a cirugía de catarata no complicada, comparando los datos de cirujanos noveles con los de cirujanos expertos. Así mismo, se compara la energía acumulada disipada (CDE, cumulative dissipated energy) del ultrasonido empleada a lo largo de la intervención entre los dos grupos, y se estudia si existe correlación con la pérdida endotelial para cada grupo.

\section{Método \\ Diseño del estudio}

Se ha realizado un estudio observacional, prospectivo y aleatorizado de pacientes sometidos a cirugía de catarata por facoemulsificación en un periodo de 4 meses en el servicio de oftalmología de un hospital universitario de tipo terciario. 
Se incluyeron 50 ojos de pacientes entre 61 y 92 años, que fueron valorados de manera consecutiva y repartidos en dos grupos homogéneos en cuanto a dificultad quirúrgica estimada, con 25 ojos operados por cirujanos adjuntos y 25 por cirujanos residentes de tercer año de este hospital, siempre tutorizados. El seguimiento, tanto antes como después de la cirugía, fue realizado por el mismo cirujano.

Se excluyeron los ojos con recuento de células endoteliales prequirúrgico $<1100$ células $/ \mathrm{mm}^{2}$ o con una fiabilidad de la determinación $<50 \%$. Los ojos con criterios de cataratas complicadas, definidas en la tabla $1, y$ aquellos que presentaron complicaciones intraoperatorias también fueron excluidos del estudio.

El recuento de células endoteliales se hace de manera sistemática previamente a la cirugía de catarata para conocer el estado del endotelio, lo que permite planificar estrategias adicionales de protección de este si fuera necesario. Esta misma medición fue repetida al mes de la operación para comparar la pérdida de células endoteliales en relación con la cirugía.

\section{Variables recogidas}

En la figura 1 se muestran todas las variables recogidas en un paciente (repitiéndose todas las mediciones y recolecciones de datos en los 49 restantes).

Los datos se recabaron con un dispositivo de miscroscopía especular de no contacto (Specular Microscope Perseus ${ }^{\circledR}$, CSO, Srl, Firenze, Italy). En cuanto a su funcionamiento, inmediatamente después de cada adquisición se despliega una pantalla resumen (Fig. 2) que muestra, por un lado, la imagen original tratada para el total de células en función de su polimegatismo (asigna un color según el área) y de su pleomorfismo (asigna un color en función del número de lados). Por otro lado, hay un menú donde se valoran estadísticamente los siguientes parámetros: área total, área medida, error estándar de la media (SEM), superficie de la célula promedio con un error dado por la desviación estándar, coeficiente de variación (CV), índice de hexagonalidad (Ex), grosor corneal, densidad e índice de fiabilidad. Esos dos últimos parámetros son los que se han tomado de referencia para realizar el presente estudio. La densidad celular se expresa en células por milímetro cuadrado. Este es el principal dato analizado en nuestro estudio y el que estima la pérdida de células endoteliales.

Por otra parte, el índice de fiabilidad indica el porcentaje de fiabilidad del análisis; la medida se da por válida cuando este parámetro es $>50 \%$.
Tabla 1. Criterios de cataratas complicadas

\begin{tabular}{|l|l|}
\hline $\begin{array}{l}\text { Catarata complicada } \\
\text { (en general) }\end{array}$ & $\begin{array}{l}\text { Catarata complicada } \\
\text { (en docencia) }\end{array}$ \\
\hline Catarata hipermadura & Ojos únicos \\
\hline Sinequias & Pacientes muy jóvenes \\
\hline Pseudoexfoliación & $\begin{array}{l}\text { Pacientes con complicaciones en } \\
\text { el otro ojo en cirugías previas }\end{array}$ \\
\hline $\begin{array}{l}\text { Bajo recuento endotelial } \\
\text { o guttas }\end{array}$ & Pacientes poco colaboradores \\
\hline $\begin{array}{l}\text { Leucomas corneales } \\
\text { Presencia de otras } \\
\text { patologías oftalmológicas }\end{array}$ & $\begin{array}{l}\text { Pacientes con importante } \\
\text { patología sistémica }\end{array}$ \\
\hline $\begin{array}{l}\text { Cámara anterior muy } \\
\text { estrecha }\end{array}$ & $\begin{array}{l}\text { Ojos muy miopes o muy } \\
\text { hipermétropes }\end{array}$ \\
\hline $\begin{array}{l}\text { Indicación de lente tórica } \\
\text { Síndrome de iris flácido } \\
\text { intraoperatorio }\end{array}$ & \\
\hline $\begin{array}{l}\text { Escasa midriasis } \\
\text { Luxaciones, } \\
\text { subluxaciones o } \\
\text { malformaciones }\end{array}$ & \\
\hline
\end{tabular}

Todos los pacientes fueron operados con la misma plataforma de facoemulsificación (Centurion ${ }^{\circledR}$ Silver System, Alcon-Novartis Pharma, USA). Todas las intervenciones de catarata se llevaron a cabo bajo anestesia intracamerular con lidocaína al $1 \%$ y a través de una incisión de $2.2 \mathrm{~mm}$. El endotelio corneal se protegió con dispositivos viscoelásticos oftálmicos: Biolon ${ }^{\circledR}$ PRIME (Bio-Technology General [Israel], peso molecular 3,000,000 Dalton) y Healon ${ }^{\circledR 5}$ (Abbott Medical Optics, Inc., peso molecular $>3,000,000$ Dalton). Se implantó en un mismo tiempo una lente ocular (CT ASPHINA $^{\circledR}$ 409M/MP ZEISS O ABBOT TECNIS ${ }^{\circledR}$ PCB00 Monofocal 1-Piece IOL) en el saco capsular. La profilaxis antiinfecciosa se realizó con povidona yodada al $5 \%$ preoperatoria y posoperatoria en la conjuntiva, junto con la administración de cefuroxima intracamerular al finalizar la cirugía. Como tratamiento posoperatorio se pautaron colirios de moxifloxacino y dexametasona. La técnica quirúrgica fue definida por el cirujano de acuerdo con las características individuales de cada caso. A las 24 horas y a la semana de la cirugía se evaluaron la estanqueidad de las incisiones, la posición de la lente, la ausencia de signos infecciosos e inflamatorios, y la posible presencia de edema 


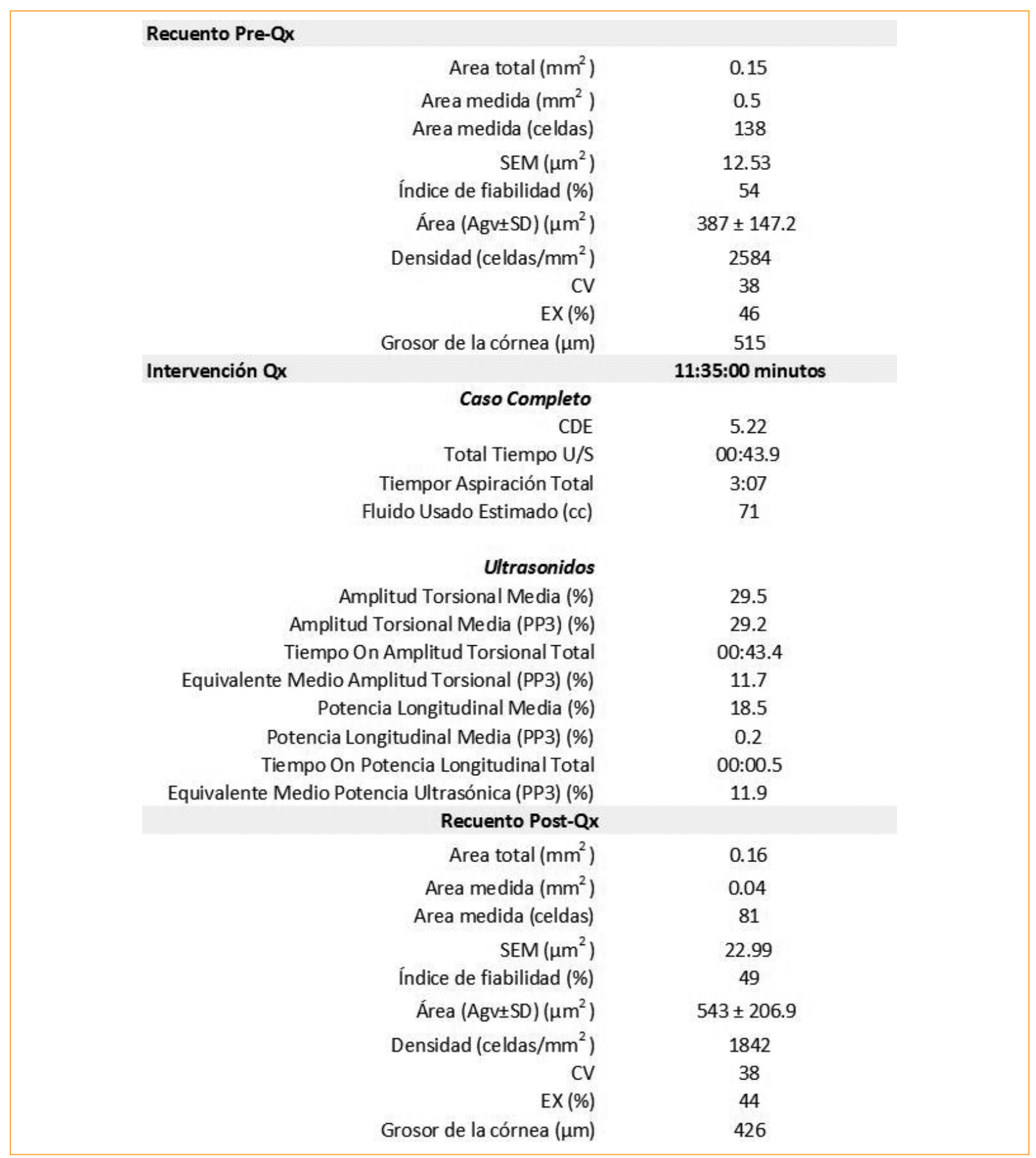

Figura 1. Variables recogidas de un paciente (se repiten todas las mediciones y recolecciones de datos en los 49 restantes). Los datos se recabaron mediante un dispositivo de microscopía especular de no contacto (Specular Microscope Perseus ${ }^{\circledR}$, CSO, Srl Firenze, Italy).

corneal. Al mes de la intervención se evaluaron la refracción y el recuento endotelial.

El equipo de facoemulsificación permite desplegar una pantalla al final de la intervención con todas las variables del caso recién finalizado (Fig. 3). De ella se tomó la CDE del ultrasonido empleada durante la intervención como dato para correlacionar con la pérdida endotelial como dato principal. Otra información que también se recogió fue el tiempo total del caso, el tiempo total de U/S, el tiempo de aspiración total, el fluido usado estimado, la amplitud torsional media (PP3), el tiempo en amplitud torsional total, el 


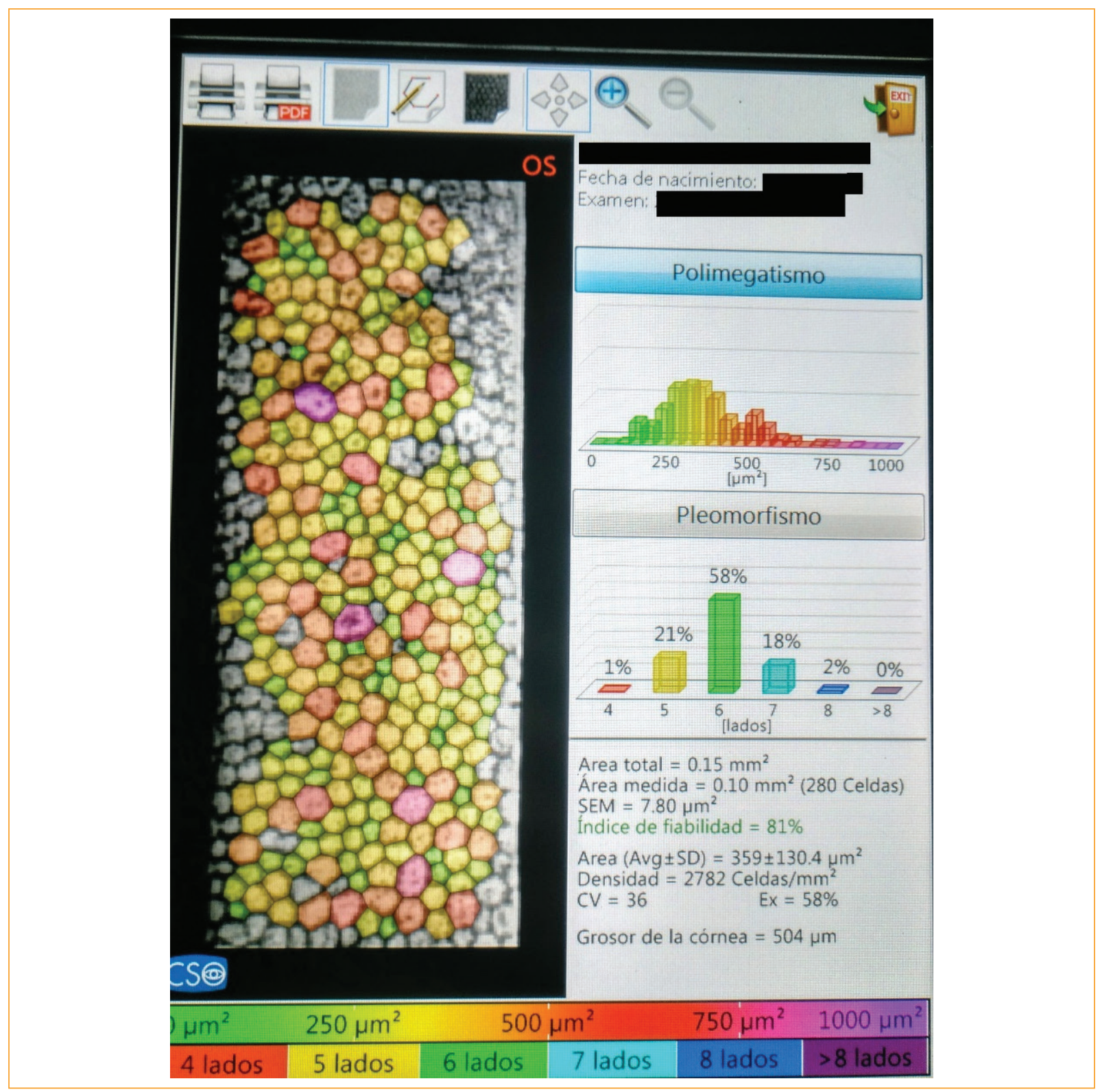

Figura 2. Pantalla resumen de adquisición del aparato (Specular Microscope Perseus ${ }^{\circledR}$, CSO, Srl Firenze, Italy). Por un lado, la imagen original tratada para el total de células en función de su polimegatismo (asigna un color según el área) y de su pleomorfismo (asigna un color en función del número de lados). Por otro lado, hay un menú donde se valoran estadísticamente los siguientes parámetros: área total, área medida, error estándar de la media (SEM), superficie de la célula promedio con un error dado por la desviación estándar, coeficiente de variación (CV), índice de hexagonalidad (Ex), grosor corneal, densidad celular (2) e índice de fiabilidad (1). Estos dos últimos parámetros son los que se han tomado como referencia para realizar este estudio.

equivalente medio de amplitud torsional (PP3), la potencia longitudinal media (PP3), el tiempo en potencia longitudinal total y el equivalente medio de potencia ultrasónica (PP3).

El estudio fue autorizado por el área de gestión científica y unidad de gestión de proyectos de investigación del hospital, y se siguieron sus normas éticas y las de la declaración de Helsinki. Se han respetado los protocolos de seguridad del hospital para acceder a los datos de las historias clínicas y preservar la privacidad de los datos personales.

\section{Análisis estadístico}

Se realizó un análisis descriptivo utilizando la mediana (percentil 50) y el rango intercuartílico 


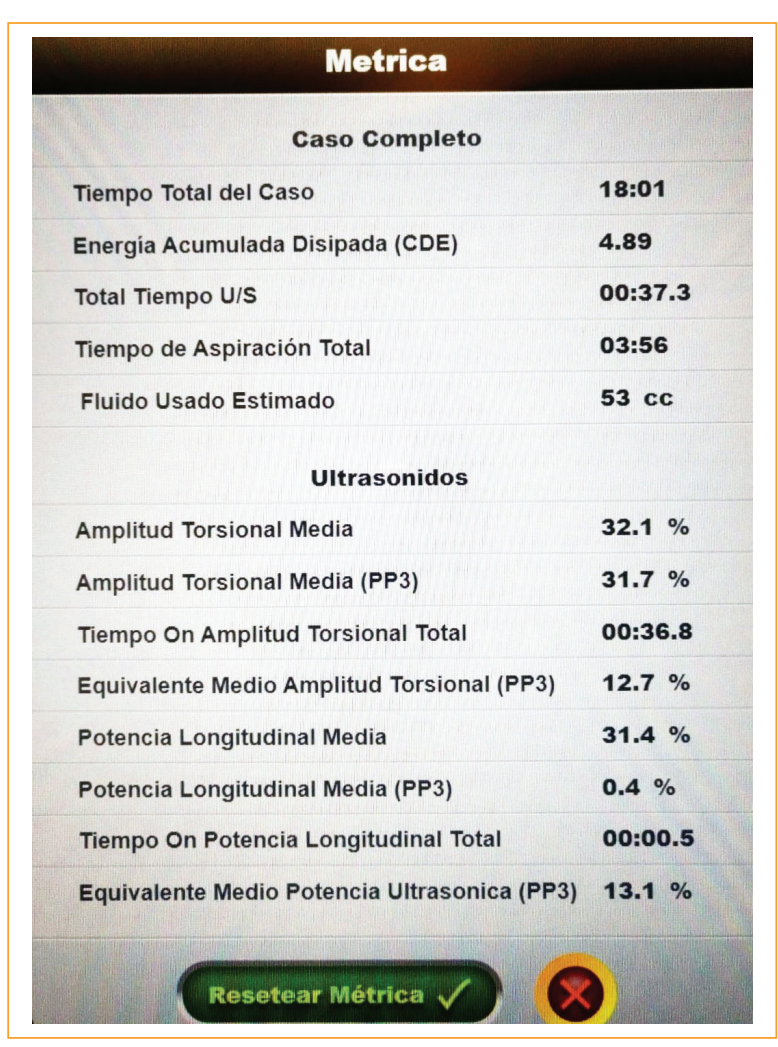

Figura 3. Pantalla resumen de los parámetros quirúrgicos tomada del aparato (Centurion ${ }^{\circledR}$ Silver System, Alcon-Novartis Pharma, US).

(percentil 25 - percentil 50) para describir las características de los pacientes. La comparación entre las principales variables según la experiencia del cirujano y la diferencia de densidad (pre-pos) se realizó con la prueba no paramétrica $U$ de Mann-Whitney y el test de la mediana.

La asociación entre la diferencia de densidades y la CDE, y la diferencia de densidades entre subpoblaciones de cirujanos (expertos y noveles), se obtuvieron mediante el coeficiente de correlación de Spearman.

El programa estadístico utilizado fue SAS versión 9.4. Se consideraron estadísticamente significativos los valores de $p<0.05$ para todos los análisis.

\section{Resultados}

\section{Características de la muestra}

Las principales características de los pacientes cuyos ojos se operaron se recogen en la tabla 2.

Los cirujanos expertos operaron ojos de pacientes con una media de edad de 72.9 años, el $88 \%$ mujeres y el $56 \%$ ojos derechos. En cuanto al grupo de ojos
Tabla 2. Características de los pacientes cuyos ojos se incluyeron en el estudio

\begin{tabular}{l|c|c|c|c|}
\hline & Mujeres & Hombres & $\begin{array}{c}\text { 0jo } \\
\text { derecho }\end{array}$ & 0jo izquierdo \\
\hline $\begin{array}{l}\text { Cirujanos } \\
\text { noveles }\end{array}$ & $17(68 \%)$ & $8(32 \%)$ & $19(76 \%)$ & $6(24 \%)$ \\
\hline $\begin{array}{l}\text { Cirujanos } \\
\text { expertos }\end{array}$ & $22(88 \%)$ & $3(12 \%)$ & $14(56 \%)$ & $11(44 \%)$ \\
\hline
\end{tabular}

operados por cirujanos noveles, la media de edad fue de 78.2 años, con un $68 \%$ de pacientes mujeres y un $76 \%$ de ojos derechos.

\section{Comparación entre cirujanos noveles y expertos}

En la muestra recogida de los ojos operados por cirujanos expertos, la mediana de la densidad celular endotelial prequirúrgica fue de 2569 células $/ \mathrm{mm}^{2}$. Tras la cirugía, el recuento endotelial objetivó una disminución de la densidad celular del $14.8 \%$, siendo el recuento final al mes de la intervención de 1965 células $/ \mathrm{mm}^{2}$. La mediana de CDE del ultrasonido durante la facoemulsificación fue de 2.61 en este grupo.

Los cirujanos noveles operaron ojos con una mediana de células endoteliales prequirúrgica de 2462 células $/ \mathrm{mm}^{2}$, perdiendo un $15.6 \%$ de la densidad celular tras la cirugía. El total de CDE del ultrasonido en este grupo fue de 4.12. En la tabla 3 se muestran las principales variables recogidas para el estudio.

En la tabla 4 se puede ver que existen diferencias entre la mediana de densidad prequirúrgica, la densidad posquirúrgica, la diferencia de densidad y la CDE entre cirujanos expertos y noveles. Sin embargo, solo se encuentran diferencias estadísticamente significativas en cuanto al uso de ultrasonidos (CDE) entre ambos grupos $(p=0.045)$.

\section{Comparación de la densidad antes y después de la cirugía y su correlación con la $\mathrm{CDE}$}

En ambos grupos hubo una pérdida estadísticamente significativa de densidad celular endotelial relacionada con la cirugía, con $p=0.0008$ y $p=0.0035$, entre cirujanos expertos y cirujanos noveles, respectivamente (tabla 5).

En los ojos operados por cirujanos noveles se demostró una buena correlación (0.577) entre la 
Tabla 3. Variables recogidas por subgrupos (cirujanos expertos y noveles)

\begin{tabular}{|c|c|c|c|c|c|}
\hline Variable & Mínimo & Percentil 50 & Máximo & $\mathbf{N}$ & Miss \\
\hline $\begin{array}{l}\text { Cirujanos expertos } \\
\text { Densidad pre (células } / \mathrm{mm}^{2} \text { ) } \\
\text { Densidad pos (células } / \mathrm{mm}^{2} \text { ) } \\
\text { Diferencia densidad } \\
\text { CDE }\end{array}$ & $\begin{array}{c}1822.00 \\
1248.00 \\
-150.00 \\
0.62\end{array}$ & $\begin{array}{c}2569.00 \\
1965.00 \\
382.00 \\
2.61\end{array}$ & $\begin{array}{c}2846.00 \\
2801.00 \\
1088.00 \\
6.93\end{array}$ & $\begin{array}{l}25 \\
25 \\
25 \\
25\end{array}$ & $\begin{array}{l}0 \\
0 \\
0 \\
0\end{array}$ \\
\hline $\begin{array}{l}\text { Cirujanos noveles } \\
\text { Densidad pre (células } / \mathrm{mm}^{2} \text { ) } \\
\text { Densidad pos (células } / \mathrm{mm}^{2} \text { ) } \\
\text { Diferencia densidad } \\
\text { CDE }\end{array}$ & $\begin{array}{c}1126.00 \\
733.00 \\
19.00 \\
0.62\end{array}$ & $\begin{array}{c}2462.00 \\
1760.00 \\
386.00 \\
4.12\end{array}$ & $\begin{array}{c}2964.00 \\
2770.00 \\
1302.00 \\
20.13\end{array}$ & $\begin{array}{l}25 \\
25 \\
25 \\
25\end{array}$ & $\begin{array}{l}0 \\
0 \\
0 \\
0\end{array}$ \\
\hline
\end{tabular}

CDE: energía acumulada disipada.

Tabla 4. Comparación entre subgrupos (expertos y noveles)

\begin{tabular}{lc} 
Variable & p \\
\hline Densidad pre (células $/ \mathrm{mm}^{2}$ ) & 0.285889 \\
\hline Densidad pos (células $/ \mathrm{mm}^{2}$ ) & 0.142934 \\
Diferencia de densidad & 0.490938 \\
CDE & 0.045642
\end{tabular}

CDE: energía acumulada disipada.

pérdida endotelial y el CDE, con significación estadística $(p=0.0025)$. Por el contrario, en los ojos operados por cirujanos expertos se obtuvo una mala correlación (0.306), sin significación estadística $(p=0.135)$.

\section{Correlación entre la diferencia de densidad novel-experto}

La tabla 6 indica que la diferencia de densidad para noveles y expertos está asociada, con una correlación de 0.589 estadísticamente significativa $(p=0.0094)$.

\section{Discusión}

El único tratamiento definitivo de la catarata es la cirugía. Con el paso del tiempo, la facoemulsificación ha ido sustituyendo a la extracción extracapsular del cristalino, hasta convertirse actualmente en la técnica más utilizada en todo el mundo 7,8 . Gran parte de las ventajas que ofrece la facoemulsificación frente a la extracción extracapsular están en relación con la mínima incisión necesaria $(1.5-3 \mathrm{~mm})^{9}$, que no precisa sutura en la mayoría de las intervenciones. Esto reduce el astigmatismo inducido quirúrgicamente ${ }^{9,10}$ y permite
Tabla 5. Comparación de la diferencia de densidad antes y después de la cirugía entre ambos grupos

\begin{tabular}{lc} 
Variable & $p$ \\
\hline Células $/ \mathrm{mm}^{2}$ en médicos expertos & 0.0008 \\
Células $/ \mathrm{mm}^{2}$ en médicos noveles & 0.0035 \\
&
\end{tabular}

Tabla 6. Correlación de la diferencia de densidad entre cirujanos noveles y expertos

\begin{tabular}{|l|c|}
\hline $\begin{array}{l}\text { Diferencia de densidad } \\
\left.\text { (células } / \mathbf{m m}^{2}\right) \text { en cirujanos } \\
\text { noveles }\end{array}$ \\
\hline $\begin{array}{l}\text { Diferencia de densidad } \\
\left.\text { (células } / \mathrm{mm}^{2}\right) \text { en cirujanos } \\
\text { expertos }\end{array}$ & $\begin{array}{c}0.58988 \\
(\mathrm{p}=0.0094)\end{array}$ \\
\hline
\end{tabular}

una pronta rehabilitación de la visión para las actividades cotidianas del paciente ${ }^{10}$. Además, se ha observado que la cirugía por facoemulsificación produce menos inflamación posoperatoria y una menor descomposición de la barrera hematorretiniana en comparación con la extracción extracapsular ${ }^{11,12}$. En cuanto a la técnica, requiere un proceso de aprendizaje lento y complejo, pero no se ha visto un aumento de la tasa de complicaciones asociado a ello ${ }^{13,14}$.

Lo que diferencia a la facoemulsificación del resto de técnicas es la fragmentación del núcleo del cristalino y la posterior aspiración de los fragmentos mediante ultrasonidos, existiendo distintas maneras para realizarlo. En este estudio, todos los cirujanos noveles operaron por la técnica «divide y vencerás», que consiste en romper el núcleo en varios fragmentos mediante el tallado de surcos con la punta del facoemulsificador (liberando con ello energía de ultrasonido) ${ }^{15}$, para 
posteriormente fracturar el núcleo ${ }^{16}$. En cuanto a los cirujanos expertos, algunos operaron con esta técnica, mientras que otros lo hicieron con la técnica de «facochop", es decir, mediante fragmentación inicial del núcleo ayudada por un instrumento cortante (chopper), prescindiendo con ello del tallado de surcos y minimizando el uso de energía de ultrasonido ${ }^{17}$. El número de cirujanos expertos que utilizaron cada técnica no se tuvo en cuenta. Tanto «divide y vencerás» como «facochop» se engloban dentro de las técnicas endocapsulares que se caracterizan por trabajar en un plano más alejado del iris, con una menor pérdida celular que las técnicas supracapsulares ${ }^{18}$. No obstante, en nuestro hospital, los noveles trabajan en un plano más anterior por miedo a dañar la cápsula posterior, asumiendo un mayor riesgo de dañar el endotelio.

De los resultados obtenidos inferimos que la técnica de facoemulsificación está asociada a una pérdida de células endoteliales, lo que implicaría que la pérdida se debe a una consecuencia real de la cirugía y no al azar.

El porcentaje de pérdida varía mucho en función de la técnica. Según ciertos autores, la técnica «divide y vencerás" produce de forma estadísticamente significativa una mayor pérdida que la técnica "faco-chop", al estar relacionada con un mayor tiempo y una mayor energía de ultrasonido ${ }^{19}$. No obstante, ambas técnicas muestran resultados y complicaciones comparables ${ }^{19}$. En este estudio se observó una mayor pérdida de células endoteliales en los ojos operados por cirujanos noveles, un $15.6 \%$, en comparación con los cirujanos expertos, un $14.8 \%$, pero sin ser esta diferencia estadísticamente significativa. Por el contrario, la correlación entre la pérdida de ambos grupos fue buena, lo que significa que los resultados en cuanto a pérdida endotelial entre unos y otros son superponibles, es decir, que en lo que se refiere a mantener una buena reserva endotelial hay muy poca diferencia entre ser operado por un cirujano novel o por uno experto. Los resultados obtenidos están en el rango de lo observado en otros trabajos ${ }^{20}$, teniendo en cuenta la escasez de literatura sobre el tema, ya que estudiarlo desde el punto de vista docente es un tema novedoso, al comparar cirujanos en formación con cirujanos experimentados.

Relacionando el total de CDE en cirujanos noveles y expertos, se demostró una diferencia significativa en el uso de ultrasonidos, lo que puede explicarse por tener los noveles una técnica menos depurada. El menor uso de energía de ultrasonido en los cirujanos expertos puede estar relacionado con el tiempo quirúrgico. En este estudio se objetivó una diferencia de 5' y 34" entre ambos grupos, siendo el tiempo medio en los noveles de 15' y 19", y en expertos de 9' y $45^{\prime \prime}$. Revisando otros estudios, Hosler, et al. ${ }^{21}$ estudiaron la evolución de la media del tiempo quirúrgico empleado por cirujanos noveles a lo largo del año académico, y concluyen que al final la mejoría es del $42 \%$. Además Randleman, et al. ${ }^{22}$ estudiaron la progresión en cuanto a competencia quirúrgica a lo largo de toda la formación de cirujanos noveles, y objetivaron que mejora progresivamente la eficiencia de la facoemulsificación y disminuye el número de complicaciones al aumentar la experiencia quirúrgica, sin alcanzar una meseta en ningún momento. Esto podría explicar por qué los cirujanos noveles son menos eficientes, pues se requiere más experiencia para aprender a optimizar el uso de ultrasonido mediante maniobras como la emulsificación a punta ocluida, el uso pulsátil de la energía, aprovechar el vacío sin ultrasonido y una fluhídrica menos prolongada, que sí que ponen en práctica los cirujanos expertos de nuestro hospital.

En relación con lo anterior, la disminución de la celularidad en el grupo de cirujanos noveles mostró estar correlacionada con el uso de ultrasonido. Teniendo en cuenta que usaron un $63 \%$ más que los expertos, los datos obtenidos para este grupo son superponibles a los resultados de estudios previos: a mayor uso de ultrasonido (CDE), más pérdida endotelial ${ }^{23}$. Por el contrario, en cirujanos expertos, la pérdida no se correlacionó con la CDE, por lo que se puede inferir que la disminución de células endoteliales en este grupo podría asumirse como la mínima esperable para la dureza nuclear media de las cataratas ${ }^{24}$. Habría que buscar otros factores de riesgo intraoperatorios que expliquen esta pérdida.

A pesar de que la técnica de «divide y vencerás» se ha convertido en el método estándar para enseñar a los cirujanos noveles a retirar de manera segura y eficiente el núcleo en la cirugía de cataratas ${ }^{16,22}$, se han realizado pocos estudios que analicen otras técnicas en cirujanos noveles. Gross, et al. ${ }^{25}$ estudiaron comparativamente los resultados obtenidos por cirujanos noveles en cuanto a CDE, tiempo de ultrasonido, beneficios y complicaciones de las técnicas «divide y vencerás» y «pop and chop», variante de la «faco-chop». Al igual que en este estudio, se excluyeron cataratas complicadas y complicaciones intraoperatorias. La técnica «pop and chop» confirmó ser segura y significativamente más eficiente que la "divide y vencerás", utilizando menos energía de ultrasonido y menos tiempo quirúrgico en cirujanos noveles. Además, los 
datos sugieren que la curva de aprendizaje de esta técnica es corta, permitiendo a los cirujanos noveles alcanzar la pericia más tempranamente ${ }^{25}$. A diferencia de la técnica «faco-chop», la «pop and chop» permite una fragmentación del núcleo bajo una visualización directa al prolapsarse parcialmente sobre la cápsula anterior del cristalino, de tal forma que es más fácil de aprender y requiere menos experiencia quirúrgica y pericia que la «faco-chop ${ }^{26}$.

En las cirugías no complicadas también se observa una pérdida de células del endotelio debido al daño mecánico intraoperatorio producido por el calor de la punta del facoemulsificador, el ultrasonido, los fragmentos del núcleo, las soluciones de irrigación, las burbujas de aire y el propio instrumental quirúrgico ${ }^{27}$. Estos factores también han podido influir en el daño endotelial sufrido tanto por la técnica de los cirujanos noveles como por la de los expertos.

Para proteger al endotelio durante intervenciones intraoculares, en 1972 Balazs, et al. ${ }^{28}$ introdujeron el uso de materiales viscoelásticos, que se clasifican en dos tipos: cohesivos y dispersivos. Los primeros destacan por mantener y estabilizar mejor el espacio y los tejidos, además de ser fáciles de inyectar y retirar de la cámara anterior ${ }^{29}$. Sin embargo, tienen tendencia a escaparse por la incisión durante la facoemulsificación, dejando el endotelio corneal desprotegido ${ }^{30}$. Los viscoelásticos dispersivos no conservan tan bien el espacio como los anteriores y son más difíciles de retirar, pero se mantienen en el interior de la cámara anterior durante la cirugía, cubriendo y protegiendo mejor las células endoteliales ${ }^{30}$.

Como características generales, todo material viscoelástico protege el endotelio de partículas y de radicales libres generados durante la intervención. Además, permite una mejor manutención de la cámara anterior y protección endotelial, disminuyendo la pérdida endotelial potencial de la cirugía ${ }^{31-33}$. Holzer, et al.$^{34}$ compararon cinco materiales viscoelásticos para evaluar la pérdida endotelial, entre los que se incluyó Healon $5^{\circledR}$. Los resultados mostraron una menor pérdida en los ojos en que se empleó Healon $5^{\circledR}$ como viscoelástico. Este es un tipo de viscoelástico viscoadaptativo (índice de cohesión-dispersión variable de 10 a 70) que se comporta de manera distinta que los clásicos cohesivos y dispersivos, de tal modo que es capaz de adaptarse de cohesivo a pseudodispersivo en función del flujo y del corte ${ }^{35}$, protegiendo mejor el endotelio y manteniendo la facilidad al retirarlo de la cámara anterior ${ }^{36}$. Los cirujanos noveles trabajaron con dos tipos de viscoelásticos, Biolon PRIME $^{\circledR}$ (cohesivo de densidad media, índice de cohesión-dispersión de 37) y Healon $5^{\circledR}$, para compensar su menor experiencia con una mejor protección del endotelio corneal. Sin embargo los cirujanos expertos trabajaron solo con Biolon ${ }^{\circledR}$. Esta menor protección del endotelio en las intervenciones realizadas por cirujanos expertos podría ser una posible causa de la pérdida objetivada de densidad celular. Se podría plantear un margen de mejora si se utilizara otro tipo de viscoelástico que fuera más estable en la cámara anterior durante la facoemulsificación ${ }^{37}$.

Nuestro estudio presenta varias limitaciones, ya que recoge un número pequeño de cirugías de cataratas, lo que podría producir una dispersión de los resultados, además de obviar otros factores que contribuirían a la pérdida endotelial. Sería recomendable realizar estudios en los que se ampliara la muestra y se tuvieran en cuenta además características fisiopatológicas específicas, como la dureza del cristalino y la profundidad de la cámara anterior. Por otro lado, sería interesante dividir la muestra en dos o más grupos según la técnica empleada en la fractura del cristalino («divide y vencerás», «faco-chop» 0 «pop and chop»).

\section{Conclusiones}

Existe una mayor pérdida de células endoteliales en los ojos operados por cirujanos noveles que en los operados por cirujanos expertos, pero la diferencia no es significativa, de tal forma que la pérdida endotelial es moderadamente superponible si atendemos a la correlación entre ambos grupos. La falta de experiencia, que implica una mayor energía de ultrasonido empleada durante la facoemulsificación, se compensa con medidas protectoras intraoperatorias, una buena tutorización y prudencia, reflejada en un mayor tiempo quirúrgico. Este mayor uso de ultrasonidos explicaría que exista una buena correlación con la pérdida endotelial en el grupo de cirujanos noveles, mientras que en los expertos la correlación es escasa, por lo que la pérdida endotelial sería atribuible a otros factores aparte del ultrasonido.

Mediante este estudio se ha podido comprobar que la docencia impartida en nuestro servicio tiene una doble vertiente: asegurar una buena técnica en las generaciones futuras de oftalmólogos y preservar la seguridad del paciente. En definitiva, mediante la revisión de la metodología propia pretendemos ayudar a mejorar la técnica quirúrgica y la docencia, lo que finalmente se traduciría en la satisfacción de la población que atendemos. 


\section{Financiación}

La presente investigación no ha recibido ayudas específicas provenientes de agencias del sector público, sector comercial ni entidades sin ánimo de lucro. El artículo e investigación forma parte de trabajos de fin de grado-master en medicina, auspiciado en la Universidad Complutense de Madrid.

\section{Conflicto de intereses}

Los autores declaran no tener ningún conflicto de intereses.

\section{Responsabilidades éticas}

Protección de personas y animales. Los autores declaran que para esta investigación no se han realizado experimentos en seres humanos ni en animales.

Confidencialidad de los datos. Los autores declaran que han seguido los protocolos de su centro de trabajo sobre la publicación de datos de pacientes.

Derecho a la privacidad y consentimiento informado. Los autores han obtenido el consentimiento informado de los pacientes y/o sujetos referidos en el artículo. Este documento obra en poder del autor de correspondencia.

\section{Bibliografía}

1. Kelman CD. Phaco-emulsification and aspiration: a new technique of cataract removal: a preliminary report. Am J Ophthalmol. 2018;64:23-35.

2. Acosta R, Hoffmeister L, Roman R, Comas M, Castilla M, Castells X. Revisión sistemática de estudios poblacionales de prevalencia de catarata. Arch Soc Esp Oftalmol. 2006;81:509-16.

3. Martínez-Soroa I, Mendicute J. Endotelio y cirugía de catarata. En: Lorente R, Mendicute J, editores. Cirugía del cristalino. Madrid: Sociedad Española de Oftalmología; 2008. p. 276-7.

4. Ruggeri A, Scarpa F, De Luca M. A system for the automatic estimation of morphometric parameters of corneal endothelium in alizarine red-stained images. Br J Ophthalmol. 2010;94:643-7.

5. Doughty MJ, Müller A, Zaman ML. Assessment of the reliability of human corneal endothelial cell-density estimates using a noncontact specular microscope. Cornea. 2000;19:148-58.

6. Maurice DM. Cellular membrane activity in the corneal endothelium of the intact eye. Cell Mol Life Sci. 1968;24:1094-5.

7. Garcés Fernández A, Hernández López I, Carmona Pérez O, Peña López L, Santana Alas E, Arencibia González D, et al. Resultados anatómicos y funcionales en la cirugía de catarata mediante la técnica de extracción extracapsular del cristalino versus facoemulsificación. Rev Cubana Oftalmol. 2011;24:64-72.

8. Adenwala A. Historia de la facoemulsificación. Dominando la facodinámica. Herramientas, tecnologías e innovaciones. New Delhi: Jaypee Brothers; 2008. p. 3-10.

9. Watson A, Sunderraj P. Comparison of small-incision phacoemulsification with standard extracapsular cataract surgery: postoperative astigmatism and visual recovery. Eye. 1992;6:626.
10. Dam-Johansen $M$, Olsen $T$. Refractive results after phacoemulsification and ECCE: a comparative study. Acta Ophthalmol. 1993;71:382-7.

11. Oshika T, Yoshimura K, Miyata N. Postsurgical inflammation after phacoemulsification and extracapsular extraction with soft or conventional intraocular lens implantation. J Cataract Refract Surg. 1992;18:356-61.

12. Pande MV, Spalton DJ, Kerr-Muir MG, Marshall J. Postoperative inflammatory response to phacoemulsification and extracapsular cataract surgery: aqueous flare and cells. J Cataract Refract Surg. 1996;22:770-4.

13. Seward HC, Dalton R, Davis A. Phacoemulsification during the learning curve: risk/benefit analysis. Eye. 1993;7:164

14. Apple DJ, Solomon KD, Tetz MR, Assia El, Holland EY, Legler UF, et al. Posterior capsule opacification. Surv Ophthalmol. 1992;37:73-116.

15. Mendicute J, Lorente R. Técnicas de fractura o cracking. En: Lorente R, Mendicute J, editores. Cirugía del cristalino. Madrid: Sociedad Española de Oftalmología; 2008. p. 662.

16. Gimbel HV. Divide and conquer nucleofractis phacoemulsification: development and variations. J Cataract Refract Surg. 1991;17:281-91.

17. Lorente R, Mendicute J, De Rojas V. Faco-chop. En: Lorente R, Mendicute J, editores. Cirugía del cristalino. Madrid: Sociedad Española de Oftalmología; 2008. p. 683.

18. Mendicute J, Lorente R. Técnicas de fractura o cracking. En: Lorente $\mathrm{R}$ Mendicute J, editores. Cirugía del cristalino. Madrid: Sociedad Española de Oftalmología; 2008. p. 652-3.

19. Watson A, Sunderraj P. Comparison of small-incision phacoemulsification with standard extracapsular cataract surgery: postoperative astigmatism and visual recovery. Eye. 1992;6:626

20. Chamorro F, Briones C, Loézar C, León A, Arancibia M, Stojanova J, et al. Pérdida celular endotelial corneal asociada a facoemulsificación y experiencia del oftalmólogo: análisis prospectivo de datos secundarios individuales. Medwave. 2018;18(6)

21. Hosler MR, Scott IU, Kunselman AR, Wolford KR, Oltra EZ, Murray WB. Impact of resident participation in cataract surgery on operative time and cost. Ophthalmology. 2012;119:95-8.

22. Randleman JB, Wolfe JD, Woodward M, Lynn MJ, Cherwek DH, Srivastava SK. The resident surgeon phacoemulsification learning curve. Arch Ophthalmol. 2007;125:1215-9.

23. O'Brien P. Fitzpatrick $P$, Kilmartin DJ, Beatty $S$. Risk factors for endothelial cell loss after phacoemulsification surgery by a junior resident. J Cataract Refract Surg. 2004;30:839-43.

24. Kreisler KR, Mortenson SW, Mamalis N. Endothelial cell loss following "modern" phacoemulsification by a senior resident. Ophthalmic Surg. 1992;23:158-60.

25. Gross FJ, Garcia-Zalisnak DE, Bovee CE, Strawn JD. A comparison of pop and chop to divide and conquer in resident cataract surgery. Clin Ophthalmol (Auckland, NZ). 2016:10:1847.

26. Chang DF. Learning phaco chop: pearls and pitfalls. New Orleans, LA: American Society of Cataract and Refractive Surgery; 2016.

27. Kohlhaas M, Klemm M, Kammann J, Richard G. Endothelial cell loss secondary to two different phacoemulsification techniques. Ophthalmic Surg. 1998;29:890-5

28. Balazs EA, Freeman MI, Klöti R, Meyer-Schwickerath G, Regnault F, Sweeney DB. Hyaluronic acid and replacement of vitreous and aqueous humor. Mod Probl Ophthalmol. 1972;10:3-21.

29. McDermott ML, Hazlett LD, Barrett RP, Lambert RJ. Viscoelastic adherence to corneal endothelium following phacoemulsification. J Cataract Refract Surg. 1998;24:678-83.

30. Koch DD, Liu JF, Glasser DB, Merin LM, Haft E. A comparison of corneal endothelial changes after use of Healon or Viscoat during phacoemulsification. Am J Ophthalmol. 1993:115:188-201.

31. Musch DC, Meyer RF, Sugar A, Soong HK. Endothelial rejection following penetrating keratoplasty using Healon or BSS. Cornea. 1990;9:305-11.

32. Alpar JJ. Use of Healon in different cataract surgery techniques: endothelial cell count study. Ophthalmic Surg. 1987;18:529-31.

33. Arshinoff $\mathrm{S}$. Los viscoelásticos en la cirugía moderna de las cataratas. En: Lorente R, Mendicute J, editores. Cirugía del cristalino. Madrid: Sociedad Española de Oftalmología; 2008. p. 502-3

34. Holzer MP, Tetz MR, Auffarth GU, Welt R, Völcker HE. Effect of Healon 5 and 4 other viscoelastic substances on intraocular pressure and endothelium after cataract surgery. J Cataract Refract Surg. 2001:27:213-8.

35. Arshinoff SA, Jafari M. New classification of ophthalmic viscosurgical devices - 2005. J Cataract Refract Surg. 2005;31:2167-71.

36. Arshinoff SA, Wong E. Understanding, retaining, and removing dispersive and pseudodispersive ophthalmic viscosurgical devices. J Cataract Refract Surg. 2003;29:2318-23

37. Martínez-Soroa I, Mendicute J. Endotelio y cirugía de catarata. En: Lorente R, Mendicute J, editores. Cirugía del cristalino. Madrid: Sociedad Española de Oftalmología; 2008. p. 281-3. 